\title{
INVESTIGACIONES
}

\section{Educación intercultural: algunas reflexiones y orientaciones para una práctica pedagógica en una escuela democrática y plural}

\author{
Intercultural education: some reflections and guidelines \\ for a pedagogical practice in a democratic and plural school \\ María Louzao Suárez, $M^{a}$ Concepción Francos Maldonado ${ }^{b}$ \\ María Verdeja Muñiz \\ ${ }^{a}$ Orientadora Educativa del IES Feijoo (Gijón). \\ marialsuar@educastur.org \\ ${ }^{b}$ Facultad de Formación del Profesorado y Educación de la Universidad de Oviedo. \\ francosconcepcion@uniovi.es, verdejamaria@uniovi.es
}

\begin{abstract}
RESUMEN
Retomar lo que ha de ser la educación intercultural y el papel que la escuela ha de jugar en ella, se nos antoja un punto de especial importancia para el profesorado en el contexto socio-político actual. Revisar lo que el currículum puede aportar en la formación para la ciudadanía intercultural puede reorientar las prácticas pedagógicas. Para esto, comenzamos haciendo referencia a la educación intercultural que defendemos y tratamos de analizar qué forma tomaría dicha aportación desde nuestro enfoque intercultural antirracista: los contenidos a enseñar y aprender; los planteamientos metodológicos más afines que habrían de impregnar la práctica y la evaluación, así como la consideración de esos otros aspectos fundamentales para la enseñanza-aprendizaje: socio-emocionales y relacionales y comunicativo-lingüísticos.
\end{abstract}

Palabras claves: Currículum, profesorado, centros educativos, inclusión, diversidad lingüística.

\section{ABSTRACT}

Taking up what intercultural education has to be and the role that the school has to play in it seems to us a point of special importance for teachers in the current socio-political context. Reviewing what the curriculum can contribute in training for intercultural citizenship can reorient pedagogical practices. To do this, we began by referring to the intercultural education that we defend and try to analyze what form that contribution would take from our anti-racist intercultural approach: the contents to be taught and learned; the most related methodological approaches that would impregnate practice and evaluation, as well as the consideration of those other fundamental aspects for teaching-learning: socio-emotional and relational and communicative-linguistic.

Key words: curriculum, professorate, schools, inclusion, linguistic diversity. 


\section{INTRODUCCIÓN}

A pesar de la trayectoria mantenida, al menos formalmente en nuestro entorno social y escolar, para dar respuesta a la diversidad con la que nos hemos ido encontrando en nuestras aulas y entornos comunitarios, los resultados, a la vista del panorama socio-político actual, no parecen ser en absoluto halagüeños. Reflexionar y revisar lo hecho en materia educativa en general y en educación intercultural en especial parece el punto de partida más obvio (Louzao, 2016) y esto conduce siempre al currículum y a la organización escolar. Acercarse al concepto de educación intercultural supone, por una parte, dejar constancia de la pluralidad de enfoques y perspectivas. También supone tener en cuenta que no hay una única definición satisfactoria. En ese sentido, y en coherencia con los planteamientos del Grupo Eleuterio Quintanilla (2000), nos identificamos con la Educación Intercultural Antirracista.

Compartimos con Besalú (2010) la idea de que los centros escolares constituyen un espacio privilegiado para incorporar la dimensión intercultural, entendida desde el enfoque intercultural antirracista, puesto que, por una parte, está presente la cultura, la reproducción, la producción y la vivencia de la cultura en sentido antropológico (en realidad, de una parte de ella, aquella que cada nación considera más relevante, más necesaria y funcional para sus miembros) y también la cultura académica, porque las escuelas e institutos gozan de una cierta capacidad para organizar y controlar el ambiente que se respira en ellos, las redes de relaciones e intercambios, las estructuras de participación y de trabajo, los recursos y materiales disponibles, las actividades a realizar y las condiciones en que se produce todo esto. Por otro lado, es necesario no caer en culturalismos, que suponen una visión estática y estereotipada de las culturas, y tener en cuenta los aspectos estructurales que conllevan discriminación y combatirlos.

El contexto en el que desarrollamos nuestra labor, Principado de Asturias, lleva ya una trayectoria consolidada en la escolarización del alumnado extranjero. Según los datos ofrecidos por el Ministerio de Educación y Formación Profesional $(2019$, p. 8) recogidos en el informe anual, la población escolar extranjera en el curso 2019-2020 constituye el $4,1 \%$ con respecto al total de la población escolar. Pueden tomarse como referencia dos trabajos de campo en centros de educación secundaria obligatoria (Díez-Gutiérrez, 2014; Verdeja, 2015) para identificar y comprender los retos educativos que conlleva la educación en contextos educativos multiculturales. Así, en el caso del profesorado, identificamos dificultades para atender a la diversidad cultural del alumnado y sus diferentes ritmos de aprendizaje, dificultades derivadas de la diversidad lingüística, un currículo en el que no hay cabida para la diversidad cultural presente en las aulas, el peso de la tradición docente del profesorado, de la carencia de recursos específicos para atender a tal diversidad, la falta de una formación específica orientada a trabajar en contextos multiculturales. En el caso del alumnado, destacamos las dificultades de adaptación a un sistema educativo diferente al de origen, inicial desconocimiento lingüístico y comunicativo, dificultades de adaptación a determinados "patrones culturales" o problemas de acceso al currículo. En el caso de las familias, los retos principales tienen que ver con problemas socioeconómicos, dificultades de conciliación para estar pendientes de la educación de sus hijos o aislamiento relacional. En definitiva, la investigación en educación intercultural en España (Rodríguez-Izquierdo, 2009), muestra que las preocupaciones de los docentes se centran, principalmente, en aspectos lingüísticos y académicos, predominando un enfoque asimilacionista de educación intercultural, si bien el profesorado muestra igualmente un interés genuino en entender la diversidad como un elemento enriquecedor para la escuela. 
En términos generales se puede decir que no existe un desarrollo normativo amplio que oriente a los centros a desarrollar proyectos educativos interculturales auténticos, demanda que se lleva publicando desde hace bastante tiempo (Grupo Eleuterio Quintanilla, 2003) y que debería tener objetivos más amplios que el desarrollo de las medidas educativas concretas para el alumnado extranjero que presenta necesidades educativas de distinto tipo como desfase curricular y/o desconocimiento de la lengua, necesidades afectivoemocionales, socio-económicas, etc. Abordar la educación intercultural supone ampliar el rango de análisis e intervención hacia los contextos extraescolares por la influencia de estos en el seno de la escuela. Interesantes aportaciones al respecto nos parecen las propuestas por Esteban (2011) partiendo del modelo ecológico de Bronfenbrenner, o las mencionadas por Gil-Jaurena (2013) al hablar de la responsabilidad del contexto socio-cultural en la consecución de los objetivos de la educación intercultural.

Precisamente es orientar la discusión hacia este ámbito la finalidad principal de este artículo: manejar un modelo que entienda la educación intercultural como un proyecto educativo sociopolítico amplio de formación de la ciudadanía, que no se puede reducir a la atención curricular de una parte de la población escolar y que, en todo caso, necesita ser urgentemente revisada para lograr una mayor cohesión social que la que se observa ahora en nuestros contextos cercanos y lejanos. Teniendo en cuenta la potencialidad de la escuela en materia de educación intercultural y la constancia, por nuestra experiencia práctica en investigación y acción en materia intercultural, de que el trabajo de actitudes y valores es una condición necesaria pero no suficiente para una verdadera educación intercultural (Louzao, 2019), nos parece pertinente abordar una reflexión acerca del currículo y de las aportaciones que, desde él, se pueden ofrecer al alumnado en aras de una educación intercultural más genuina.

Centraremos, en primer lugar, los puntos clave sobre los que vamos a abordar la cuestión, y que luego nos remitirán a unas orientaciones prácticas. A saber, a) exponer la idea de currículum que defendemos y su relación con lo intercultural; b) explicitar qué modelo de educación intercultural asumimos, coherente con lo anterior y su relación con una formación para la ciudadanía; c) qué concepción del alumnado manejamos; d) cómo podemos concretar todo lo anterior en orientaciones para la práctica que arrojen luz en los difíciles tiempos que (nuevamente) vivimos, y esto desde la dificultad para transformar prácticas y organizaciones que observamos (y padecemos diariamente) como trabajadoras en los centros escolares. De esta forma, tendremos como referente lo ideal para encajarlo en lo real, entendiendo la mejora como un desarrollo profesional docente alejado de la rendición de cuentas (Merchán, 2012), que no es sino la política educativa que presiona a los centros en la búsqueda de resultados y que provoca la pérdida de identidad y motivación profesionales (Merchán, 2013).

\section{BREVE REPASO POR LA NORMATIVA QUE RECOGE LA ATENCIÓN EDUCATIVA AL ALUMNADO DE INCORPORACIÓN TARDÍA EN ASTURIAS}

Una parte muy importante del alumnado extranjero (inmigrante o no) que tenemos escolarizado en nuestros centros es considerado, en algún momento de su trayectoria escolar (sobre todo en los inicios) como alumnado de incorporación tardía. La normativa general para la escolarización de este alumnado que existe en Asturias, se nutre de la 
normativa estatal y específica sobre alumnado con necesidad específica de apoyo educativo y, más concretamente, de quienes presentan necesidades educativas por provenir de otros países, o por presentar desconocimiento del español o tener escasos conocimientos curriculares básicos (leyes orgánicas, reales decretos y decretos de currículo de las distintas etapas, así como las Resoluciones por las que se aprueban las instrucciones que regulan la organización y el funcionamiento de los centros escolares de nuestra comunidad autónoma). En Asturias se comienza a sistematizar la atención a este alumnado a partir del año 2000, con la elaboración del documento "La atención a alumnos inmigrantes en la Comunidad Autónoma de Asturias”, que incluye el Plan de Acogida Socio-Lingüística para escolares, la creación de las Aulas Temporales de Inmersión Lingüística a partir del curso 20042005 y otra serie de propuestas (muy escasas) para el trabajo con la comunidad escolar y el entorno desde una perspectiva intercultural. En el curso 2006-2007 se incorporan dos nuevas medidas, la tutoría de acogida y las aulas de acogida y de acceso al currículo.

Este desarrollo normativo se consolida el curso 2017-2018 con la Circular de 14 de noviembre de 2017 sobre la atención educativa al alumnado de incorporación tardía, para centros docentes públicos y centros docentes concertados y cuyas principales medidas serían: la flexibilización del periodo de escolarización; el Plan de Trabajo Individualizado para el alumnado; la tutoría de acogida; las Aulas de Acogida y de Acceso al Currículo y las Aulas Intensivas de Inmersión Lingüística. Esta circular se consolida como documento normativo de referencia en el presente curso 2018-2019, en el que se sigue echando en falta una propuesta de educación intercultural que vaya más allá de la atención específica (y desde un determinado enfoque) de una parte de la población escolar.

Tal como aparece recogido literalmente en este último documento mencionado, la Circular sobre la atención educativa al alumnado de incorporación tardía al Sistema Educativo Español para los centros públicos y para los centros concertados, para el curso 2018-2019, se entiende por alumnado de incorporación tardía al sistema educativo aquel que se incorpora a los centros educativos a partir del $2^{\circ}$ curso de Educación Primaria hasta el último curso de la escolarización obligatoria y que, por proceder de otros países, presenta un desconocimiento de la lengua española o un desfase curricular importante debido a escolarizaciones irregulares anteriores o nulas. Las medidas de acogida que se establecen en esta circular se aplicarán al alumnado que se haya incorporado recientemente al sistema educativo, entendiendo el plazo de tres cursos académicos, desde su matriculación, como el máximo para acogerse a algunas de ellas. Si pasado este tiempo el alumno o alumna necesita más apoyo, se le proporcionarán otras medidas de atención a la diversidad que estén recogidas en el PAD (Plan de Atención a la Diversidad) del centro.

Retomando la última idea mencionada en el párrafo anterior, la escasa presencia de contenidos y objetivos interculturales se puede observar cuando, al leer el documento, se menciona únicamente como objetivo general de la atención educativa al alumnado con necesidades educativas derivadas de su incorporación tardía, garantizar una respuesta educativa de calidad y ajustada a sus características personales, en función de su diversidad cultural o de otras necesidades de carácter personal, familiar o social. Entre los objetivos específicos sí se explicita que hay que promover la educación intercultural de la población escolar y la necesaria coordinación con el entorno socio-educativo, por cuanto ofrece recursos que también dan respuesta a las necesidades que presenta dicho alumnado. Pero, cuando entramos en profundidad al análisis de las medidas, no encontramos las líneas directrices básicas para un currículum intercultural que vayan más allá de promover el 
respeto, la comunicación y comprensión mutua entre todo el alumnado con independencia de varios factores, entre ellos el origen cultural. Como sabemos y hemos mencionado previamente, condición necesaria pero no suficiente para una verdadera educación intercultural de calidad.

Partiendo de la adopción del modelo intercultural antirracista que adoptamos, no se puede reducir a la atención al alumnado extranjero ni que la labor de la escuela sea únicamente la de poner en marcha medidas organizativas dirigidas a reducir el fracaso escolar de los recién llegados y favorecer su asimilación (Besalú, 2008). Es un modelo que requiere trabajar con el conjunto de la comunidad educativa y no reducirse a poner en marcha una serie de medidas dirigidas únicamente al alumnado extranjero por el mero hecho de serlo. Nuestra experiencia práctica, además, nos demuestra que el alumnado cuenta con muy diferentes experiencias previas de escolarización, no por ello irregulares (tienen multitud de aprendizajes adquiridos que hay que aprovechar) y que hablar de educación intercultural tiene que incluir necesariamente aprovechar el multilingüismo presente en las aulas (incluidas las variedades del español en otras partes del mundo).

\section{LA EDUCACIÓN INTERCULTURAL QUE DEFENDEMOS}

Ya hemos descrito más ampliamente (Louzao, 2019) adónde nos ha conducido la revisión y elección de nuestro modelo de educación intercultural antirracista. En todo caso, retomamos las esencias fundamentales de nuestro posicionamiento para luego entender más fácilmente por qué manejamos una idea y no otra de currículum y por qué nuestras orientaciones para la práctica toman un camino y no otro.

Brevemente, recordaremos que dejamos atrás la consideración de la realidad social como meramente multicultural, bien porque como explicaba Touraine (2001) el mero contacto entre culturas no significa necesariamente más que eso. Es más, preponderar esa obviedad y no ir más allá permite al neoliberalismo reducir y controlar a las minorías étnicas (Bauman, 2003), convirtiéndose en una estrategia de dominación (Walsh, 2010) y no erradica el racismo (Grupo Eleuterio Quintanilla, 1996, 2000).

Recalcamos, pues, que partimos de un enfoque socio-crítico de educación intercultural antirracista que remite a la idea de una formación para todos y todas, en el seno de una institución, la escolar, que ha de replantearse su tradicional función política de formar a una ciudadanía culturalmente homogénea (Bolívar, 2004). En palabras de Walsh (2010), una educación intercultural crítica ha de ser un proyecto político global de la sociedad, cuya finalidad principal sea la de reconstruir constantemente esa sociedad, cuyo funcionamiento actual sigue perpetuando la desigualdad, la discriminación y la dominación de unos grupos culturales sobre otros.

Si entendemos un modelo socio-político de convivencia y de formación para la ciudadanía cuya finalidad principal es la transformación social para el pleno desarrollo del ser humano, visibilizando las barreras que perpetúan la desigualdad y la injusticia social, actuando contra ellas y creando las condiciones para la participación ciudadana en el espacio público a través de un proceso educativo y de formación que dote a las personas de los conocimientos, actitudes y habilidades personales necesarias para participar con garantías y reconocimiento en dicho espacio público (Louzao, 2019, p. 47), asumimos la naturaleza socio-política de la escuela y del currículum que tiene que formar para una 
ciudadanía intercultural que no solo trabaje el desarrollo de valores y actitudes, sino que ofrezca herramientas de pensamiento (formación e información) que doten de sentido crítico a esos valores y actitudes como medio de transformación social y de participación en la vida pública y que se dirija al conjunto de la comunidad educativa.

Estaríamos hablando de una "ciudadanía intercultural" en palabras de Pérez Tapias (citado en Bolívar, 2004, p. 21) en los siguientes términos: una ciudadanía que no se puede construir sobre diferencias étnicas y culturales sino a través del derecho de comunicación y de participación que nos asiste como sujetos políticos (Habermas, citado en Bolívar, 2004, p. 24).

\section{NOCIONES GENERALES SOBRE CURRÍCULUM Y EDUCACIÓN INTERCULTURAL}

Todo lo incluido en el apartado anterior nos conduce a aclarar nuestra idea de currículum, una que ha de aglutinar los ingredientes anteriores y que tenga carácter transformador. Quizá el punto de partida necesario sea precisar qué entendemos por tal, dada la tendencia general a identificarlo con las prescripciones administrativas. Aquí, por el contrario, compartimos la siguiente definición de Romero (2018):

(...) al hablar del currículum no me refiero únicamente al programa de estudios y sus contenidos, o las decisiones de diversa índole que convergen en la planificación didáctica. La acepción que tengo en mente contempla -además de lo prescrito, escrito, diseñado o deseado- la ardua dialéctica entre intenciones y realidad. Esto es, en el término englobo también el currículum "abrazado" en la práctica, el representado o llevado a cabo en las interacciones entre profesores y alumnos a través de un incierto juego de interpretaciones, negociaciones, actuaciones, rituales, controles, acomodaciones, omisiones, resistencias o rechazos que lo reconstruyen en el espacio físico e institucionalmente estructurado de las aulas, modelando el tipo de experiencias "educativas" - expresas y tácitas- ofrecidas o negadas de facto a los discentes (pp. 105-106).

El siguiente paso -seguimos apoyándonos en el interesante análisis del autor recién citado- es desnaturalizar la producción y reproducción de esa selección arbitraria de cultura, atravesada por unas líneas de inclusión y exclusión. Esto es, impugnar el falso sentido común que asume el currículum como una "esencia epistemológica" irreductible, ocultando que es una construcción social e histórica controvertible, susceptible de ser revisada críticamente desde su misma raíz. ¿Qué selección y organización del conocimiento escolar puede acercarnos a una idea, no retórica, de cultura para todas y todos, incluyendo en ese "todos" no sólo a los "similares" a "nosotros" sino también a los "diferentes" en su alteridad? En otras palabras, ¿qué cultura para un "nosotros" no lastrado por sesgos nacionalistas, etnocéntricos, de género o clase social?

Si hablamos de formar ciudadanía intercultural para una sociedad democrática, la ciudadanía, es decir, nuestro alumnado en formación debería tener la oportunidad de aprender lo que esa forma de vida significa y cómo se puede practicar (Dewey, citado en Apple y Beane, 2005, p. 23). Y somos nosotras, las educadoras, las que tenemos que construir la escuela como un espacio democrático de formación para la ciudadanía. Los 
anteriores autores se refieren a dos líneas de trabajo que habría que seguir para alcanzar dicho objetivo:

Por una parte, la escuela debe reconstruirse como un espacio para la participación igualitaria, dando voz a todos y todas (Susinos y Ceballos, 2012) y garantizando la posibilidad de aprender a ser ciudadano/a, porque esa es una condición por aprender (Romero, 2005).

De forma complementaria, Apple y Beane (2005) nos recalcan la necesidad de un currículo democrático, para nosotras socio-histórico: un currículum que ofrece alternativas al pensamiento hegemónico, presentado como "neutral" y que entiende al alumnado como un receptor pasivo de conocimientos para reformularlo, ya que "las disciplinas de conocimiento no son simplemente el "conocimiento académico oficial" que el alumnado tiene que aprender; son fuentes de comprensión e información que se pueden poner en relación con los problemas de la vida" (p. 34). Aquí es importante valorar "qué papel podría jugar el pensamiento generado por el profesorado" (p. 38) y sumar ambos aspectos para " (...) reconstruir el conocimiento dominante y utilizarlo para ayudar a los que tienen menos privilegios en esta sociedad (...)” (p. 36). Por su parte, Koustourakis et al. (2016) nos recuerdan nuevamente, fruto de su investigación, la relevancia de tener en cuenta el capital cultural familiar en el éxito educativo de las jóvenes generaciones y el papel que juega el conocimiento escolar en la construcción de dicho capital. Constantes estudios siguen poniendo de manifiesto, por una parte, que ciertos grupos (normalmente los que tienen mayor riesgo de no alcanzar el éxito educativo) están silenciados en la escuela y, por otra, que el colectivo de alumnado inmigrante tiene mayores tasas de fracaso escolar que el alumnado autóctono (Huguet y Navarro, 2006; Jiménez, Sánchez, García y Cotrina, 2011). Por otra parte, cabe preguntarse si todo estudiante inmigrante tiene más posibilidades de fracaso escolar que un estudiante autóctono. Responder a esta cuestión no deja de ser una tarea compleja porque, incluso, hay diferencias entre los propios estudiantes inmigrantes. Para reflexionar en torno a estas cuestiones lo hacemos acercándonos al modelo ecológico de Ogbu (1993) que plantea que existen diversos factores explicativos del fracaso o éxito escolar y que éstos se sitúan, no solo en el desajuste en los estilos de comunicación o de formas de relacionarse con los saberes de la escuela, sino en las estrategias sociales y económicas desplegadas con respecto a la escuela por cada minoría o colectivo en función de la estructura de oportunidades existentes en la sociedad. Por consiguiente, se precisa una explicación a nivel múltiple (que conjugue los aspectos micro o interpersonales que se dan en las aulas y los macro o socioeconómicos), que estudie los factores (sociales, estructurales y culturales) en combinación, de un modo ecológico, y que, además, dé cuenta de por qué algunas minorías étnicas no presentan altas tasas de fracaso escolar y otras sí (Abajo y Carrasco, 2011, p. 74).

\subsection{CURRÍCULUM INTERCULTURAL Y CONTENIDOS}

El marco teórico que venimos planteando, que se fundamenta en principios de investigaciónacción, desde el enfoque intercultural antirracista, insta a reflexionar sobre el currículum, las metodologías, los aspectos socioemocionales y relacionales. A esta reflexión dedicaremos los apartados siguientes.

Un currículum intercultural apela, en primer lugar, al conocimiento que pensamos construir con todo el alumnado: qué enseñar y qué aprender. Apple y Beane (2005) 
entienden que un currículum democrático ha de ofrecer al alumnado una gran variedad de información, "los problemas de la vida" (p. 34) que nos atañen a todos y todas, para manejar y reinterpretar; una información que permita valorar las opciones existentes y que permita tomar decisiones ante las diferentes alternativas de pensamiento existentes. Ya en otros contextos hicimos alusión a la necesidad de organizar el currículum en torno a problemas sociales de la vida como un elemento clave para un currículum intercultural. Hay múltiples experiencias al respecto en escuelas democráticas avaladas por sus excelentes resultados en el proceso de aprendizaje y formativo de su alumnado.

Si nos atenemos a nuestra más inmediata y concreta realidad, el análisis del currículo que manejamos en nuestra práctica diaria apunta a propuestas de trabajo mínimamente conectadas con un enfoque intercultural (Verdeja y González, 2018) pero todavía sin superar las limitaciones de un "currículum como hecho" e, incluso, de un "currículum como práctica", si bien acercarnos mínimamente a esta última acepción supone avanzar hacia un currículum socio-histórico por cuanto que las prácticas en sí pueden favorecer contextos más democráticos de aprendizaje y de formación. Numerosas investigaciones siguen poniendo de manifiesto la escasez de contenidos en el currículum que destaquen el valor de otras culturas no hegemónicas o bien su inclusión en el currículum desde una perspectiva folclórica (Torres Santomé, 2011) que, lejos de avanzar hacia lo intercultural, refuerzan los estereotipos y los prejuicios existentes sobre los grupos minoritarios. Sería un currículum que habría de incluir necesariamente lo que Esteban y Vila (2013) definen como los "fondos de conocimiento e identidad" del alumnado, con independencia de su origen.

Para poder avanzar y reconstruir dicho currículo "oficial" podríamos comenzar planteándonos varios interrogantes a los que podríamos responder seleccionando los contenidos a trabajar, la metodología a utilizar y las propuestas de cambio a publicitar, tales como: ¿Sobre qué se debería hablar y pensar en la escuela? ¿Sobre qué se debería leer, escribir e investigar en la escuela? Tomando como referencia estos interrogantes, que ya orientan claramente hacia lo que habría que trabajar en las aulas, proponemos a continuación algunas sugerencias específicas para las áreas comunes, que son aquellas que cursa todo el alumnado.

\subsection{CURRÍCULUM INTERCULTURAL Y METODOLOGÍA}

Un currículum intercultural no se puede reducir únicamente a los contenidos sino también ha de considerar la metodología. Sin embargo, entender el currículum desde esa perspectiva socio-histórica nos impele a entender que la metodología, por sí sola, no transforma el currículum ni la organización escolar. No se puede entender la metodología sin los contenidos que la sostienen. Es cierto que existen ciertas formas de hacer a priori más inclusivas que otras, pero es precisamente vaciarla de contenido lo que favorece la permanencia de ciertos contenidos y sus planificaciones educativas históricamente asentadas en las aulas y que, a la postre, perpetúan el currículum como hecho. Pongamos algunos ejemplos: trabajar por proyectos contenidos poco significativos e inmersos en una dinámica calificadora como la que tenemos, reduce el potencial de la metodología a ciertos momentos que no tienen su generalización fuera del aula, por cuanto orientan el trabajo escolar y extraescolar a dominar dichos contenidos de manera repetitiva y memorística que, a la postre, es lo que define la calificación obtenida y que se olvidan, al menos, con 
la misma rapidez con la que se adquirieron. Su capacidad transformadora es temporal e incompleta. Más ejemplos: la organización del aula por rincones y la asamblea como metodología de aprendizaje cooperativo y dialógico se convierten, rápidamente, si nos descuidamos, en espacios y tiempos vacíos de contenidos relevantes de aprendizaje, por cuanto tienden a introducir sistemáticamente el currículo oficial de cursos posteriores o bien se dejan al libre albedrío de los niños y niñas del aula, que han de rellenar el tiempo de trabajo en aras de una mal entendida libertad y creatividad.

Con todo esto, entendemos que una metodología interesante desde la perspectiva del currículum que manejamos y que es coherente con una perspectiva intercultural es aquella que:

- Se desarrolla a partir de los interrogantes que nos interesa plantear en cada momento y que tiene en cuenta a todo el alumnado. Una metodología que ni se plantea dar cuenta de la operativización de dichos interrogantes en términos cuantitativos y que renuncia a organizar el trabajo del aula tomando como referente el teaching to the test que tan prolíficamente se nos invita a incluir. Dicha orientación no puede ser una realidad en nuestra práctica por cuanto perjudica al alumnado que ya está en riesgo de exclusión (Romero, 2018).

- Una metodología que organiza los espacios de aprendizaje y los agrupamientos del alumnado de tal manera que se promueva un aprendizaje genuinamente cooperativo (Díaz-Aguado, 2004) y, mejor aún, colaborativo (Escarbajal, 2010) y con él, la construcción negociada y compartida del conocimiento. Sin olvidar los estilos de aprendizaje y su adaptación a diversas metodologías. Estos agrupamientos superan cualquier forma de mixture o streaming (Calatayud, 2018) y avanzan hacia otros más flexibles y genuinamente inclusivos. En definitiva: aprendizaje dentro y fuera del aula, todos y todas juntos, con adultos y adultas expertos, para pensar y aprender a decidir conjuntamente sobre temas de nuestro interés.

- Una metodología que se inspira en la investigación a través de distintas fuentes, que sacan a la luz las diferentes cosmovisiones que sobre el conocimiento existen; una metodología que promueve la participación: en clase se lee y se escribe, se habla y se escuchan todas las voces posibles. Las educadoras somos las responsables de facilitar el acceso al conocimiento y a las fuentes a todo el alumnado, especialmente al que tiene más dificultades para ese acceso.

- Una metodología de aprendizaje que propone actividades interesantes para el alumnado, con distintos ritmos de trabajo y niveles de profundización y que, por tanto, evita la exclusión de una parte del grupo en su participación, evita la repetición y sugiere distintas formas de resolución a las mismas: no se trata de realizar muchas veces la misma actividad sino de despertar la curiosidad del alumnado por dar respuestas. Esta forma de trabajar debe estar provista de distintos recursos, no solo los escritos, debiendo estar todos ellos libres de sesgos racistas, machistas y/o discriminatorios por la razón que sea. Es evidente que el libro de texto, como fuente de conocimiento, ha de ser sometido a valoración, evidentemente, también los destinados específicamente al alumnado extranjero no hispanohablante (véase al respecto la interesante investigación realizada por López-Jiménez, 2018). Ningún material utilizado puede ser una fuente para perpetuar la desigualdad ni el pensamiento hegemónico dominante. 
Para ir concluyendo, diremos que es una metodología que valora el conocimiento aprendido por el alumnado, pero para "elevarlo" a un mayor nivel de complejidad y abstracción, promoviendo su movilización para una mejor comprensión de la realidad social que nos rodea. El alumnado posee conocimientos que hay que sacar a la luz para dotarlos de prestigio y, en todo caso, ayudar a que los amplíe en forma y perspectiva.

Igualmente, es una metodología que demanda al profesorado someter a revisión constante sus propias ideas y principios; una investigación-acción participativa y transformadora, cuya esfera de acción sea el espacio interno y externo al centro porque:

(...) si aspiramos a la mejora, el propósito de la formación deberá ser dilatar racionalmente, por una parte, nuestro sentido de la realidad (la capacidad de explicar por qué las cosas son como son y por qué acostumbramos a contemplarlas como lo hacemos), rehuyendo las invitaciones a un voluntarismo ingenuo que suele ser presa fácil de las inercias estructurales y las rutinas cotidianas. Y, por otra parte, nuestro sentido de la posibilidad (la facultad de pensar que las cosas podrían ser de otro modo y, llegado el caso, desear que lo fuesen (Romero, 2018, p. 15).

\subsection{CURRÍCULUM INTERCULTURAL Y EVALUACIÓN}

Otro elemento fundamental sobre el que hay pensar es la evaluación. Es fundamental adoptar un enfoque intercultural en la evaluación porque dicho enfoque "recognizes and values the cultural and linguistic diversity of a society" (Schmelkes, 2018, p. 3). Esta autora también menciona que dicho enfoque nos servirá como herramienta para poder sacar a la luz y detectar las desigualdades que afectan a los colectivos en riesgo de exclusión y/o con representatividad minoritaria (entre ellos el colectivo de alumnado inmigrante en su mayoría), al mismo tiempo que nos permitirá la inclusión de estos en el marco curricular y escolar.

Sabemos que la evaluación es un componente esencial de la institución escolar, que condiciona de tal forma la vida escolar que es muy discutido y discutible, controvertido y polémico, todo lo que se mueve alrededor de ella (Gimeno, 1996). Es evidente, sigue señalando Gimeno, la influencia que la evaluación tiene en todo el aprendizaje y en la enseñanza y tiene formas muy diferentes de entenderse, tanto en sus objetivos y finalidades como en sus procesos. Si atendemos a sus aportaciones, consideramos que una evaluación coherente con un enfoque intercultural conllevaría adoptar un enfoque de esta a nivel de centro que pase de la manida cultura del examen a una otra de la evaluación, entendida ésta, como una oportunidad para la mejora, para el desarrollo profesional docente y para la inclusión educativa (Ávila, Calatayud, Cantón, Castillo y Zaitegui, 2010). ¿Cuáles serían en el centro las señas de identidad de este modelo de evaluación? A saber, las que orientan la reflexión desde la cultura evaluadora que manejamos hasta las prácticas concretas en las que ésta se operativiza. Entendemos que:

- La escuela actual intercultural necesita otra cultura evaluadora. Para ello, un proyecto de educación intercultural tiene que proponer revisar e investigar sobre nuestra práctica y sobre el funcionamiento de la organización escolar para sacar a la luz nuestras prácticas excluyentes y reconducirlas.

- El trabajo del profesorado inmerso en esa nueva cultura evaluadora es un trabajo que despeja dudas y elimina suspicacias acerca de la observación de lo hecho y 
pensado por otros y otras. Entiende que las aulas, como uno de los posibles, es un espacio abierto y de aprendizaje compartido.

- Las programaciones didácticas han de diseñar situaciones genuinas de aprendizaje, no situaciones artificiales de repetición de unos contenidos seleccionados en función de evaluaciones estandarizadas externas que orientan la enseñanza solo en una determinada manera.

Como viene siendo habitual, estas orientaciones generales ayudarán a todo el alumnado y, más específicamente, al alumnado extranjero que requiera medidas educativas complementarias. Al respecto, partiendo de esas líneas generales, proponemos estas otras más específicas con respecto al alumnado extranjero y las limitaciones que observamos en la práctica diaria con respecto a la evaluación formal:

- Es necesario incluir en la normativa que regula la evaluación de los aprendizajes del alumnado la idoneidad de flexibilizar los tiempos para evaluar y, muy concretamente, sustituir las dinámicas calificadoras por otras dinámicas evaluadoras más positivas para el progreso del alumnado. Un alumno o alumna que desconoce el español porque acaba de llegar a nuestro país, pero que habla varias lenguas y que puede haber tenido experiencias escolares previas tan exitosas como el alumnado que vivió siempre en nuestro país, no puede ser calificado como incompetente (lingüísticamente, por ejemplo) a final del trimestre. La administración y los centros han de hacer un mayor esfuerzo que completar esta calificación con unas meras observaciones (que en muchos casos no dicen nada) y que, en todo caso, no tienen validez en el imaginario colectivo del alumnado (sabemos que la nota es lo que importa).

- Es necesario, también, dar una vuelta a los instrumentos de evaluación que utilizamos, teniendo en cuenta que lo que el alumnado sabe lo puede demostrar de muchas maneras y que dichos instrumentos hay que (se puede) adaptarlos a las dificultades iniciales en la comunicación.

- Somos conscientes de que esto no se puede llevar a cabo si no pensamos nuestros tiempos como profesorado de otra manera. Es cierto que nuestras condiciones de trabajo actuales son difíciles, con mucha diversidad a la que atender y poco tiempo para ello. Precisamente por esto, diseñar un proyecto educativo intercultural (como una pequeña parte del proyecto educativo del centro) permitiría sistematizar un plan de trabajo que optimizaría el tiempo y que ayudaría, partiendo de una cultura de la evaluación participativa, en la que el profesorado comparte y se apoya mutuamente. Si sabemos cómo adaptar instrumentos, si utilizamos un modelo de seguimiento y evaluación que contenga información para la mejora y no una calificación, el grado de satisfacción y compromiso del profesorado aumentaría mucho y también lo haría la atención educativa que recibe nuestro alumnado extranjero. Hay que entender que el desajuste personal cuando, chicos y chicas, niños y niñas, que eran escolares exitosos en su lugar de origen, son inicialmente considerados como incompetentes por no haber contado con el tiempo suficiente para poder demostrar eso que ya saben. 


\section{ASPECTOS SOCIO-EMOCIONALES, COMUNICATIVOS Y RELACIONALES QUE ESTÁN PRESENTEN EN EL APRENDIZAJE Y EL CURRÍCULUM}

Hablar de currículum y organización escolar para una educación intercultural como la que defendemos no puede olvidar la importancia, ya significada anteriormente, de considerar esos otros aspectos que influyen tan decisivamente en la enseñanza y el aprendizaje y que impregnan todo el currículum, a saber: los aspectos afectivo-emocionales, socio-relacionales y comunicativo-lingüísticos. Ya hemos dejado de manifiesto que la educación intercultural que defendemos supone entender la formación ciudadana desde una perspectiva amplia y ello supone entender también que hemos de desarrollar un conjunto de actuaciones dirigidas específicamente a dar respuesta a las necesidades educativas que pueda presentar, en este caso, el alumnado extranjero, y a las que nos hemos referido en el apartado de la introducción. Es obvio que no se pueden abordar ambas cuestiones por separado, por lo que sobre estos últimos aspectos escribimos, pero no pensamos, desligados de todo lo que tiene que ver con el currículo escolar.

Desde estas consideraciones, un punto de partida es destacar que la categorización de alumnado de incorporación tardía no puede considerarse una etiqueta homogénea, pues bajo esa denominación se engloba a alumnado muy diverso: por su origen geográfico, por sus circunstancias familiares y socioeconómicas, por sus historias de vida, por su escolarización previa, por sus expectativas, etc. Igualmente, que la lengua, como instrumento de comunicación fundamental en la escuela, y su desconocimiento, es percibida por la mayor parte del profesorado como una dificultad muy importante para la incorporación al currículo del alumnado extranjero que la desconoce. Como profesorado hemos de tomar decisiones muy importantes acerca de cómo vamos a conseguir que la aprenda al mismo tiempo que aprende los contenidos curriculares.

Igualmente, tenemos en cuenta la diversidad lingüística del alumnado como otro elemento favorecedor de la equidad y la cohesión social en la escuela. Es de sobra conocido el papel de la comunicación y de las lenguas como requisitos, no sólo para la comunicación, sino también para el aprendizaje, de ahí que tengamos que prestar atención a esto, y todo en un contexto de amplia diversidad lingüística, tanto por parte del alumnado extranjero como también del autóctono.

Cualquier factor que afecta, tanto a su proceso de adquisición como de desarrollo, va a interferir, no sólo en el plano personal sino también y especialmente en el plano académico (Fernández Enguita, 2001). Si sabemos, además, que actualmente el alumnado extranjero presenta una tasa mayor de fracaso escolar que el alumnado autóctono, es obvio la atención que hemos de añadir en el alumnado extranjero que desconoce inicialmente el código de acceso al currículo, así como otras consideraciones relevantes sobre el aprendizaje de segundas lenguas, que orientarán las ayudas que habremos de ofrecer, individualizadas a cada situación, para que todo el alumnado consiga un desarrollo comunicativo y lingüístico que le permita progresar con éxito en el sistema escolar.

\subsection{ASPECTOS AFECTIVOS Y EL CURRÍCULUM}

Un aspecto para tener en cuenta en todo momento es el emocional. Si las emociones influyen en nuestros pensamientos, ¿cómo influirían en una situación de cambio? Este alumnado (y aquí generalizamos, dado que, independientemente de las circunstancias personales, 
vivir en un nuevo contexto supone una cierta inquietud, incluso en el caso de vivirlo con euforia) se encuentra en una situación de desarraigo, de choque cultural (Massot, 2003) y es preciso que el profesorado, el centro, la comunidad educativa, ofrezca un ambiente cálido. Además, en el campo de las lenguas, desde hace décadas son conocidas las investigaciones, entre otros autores, de Krashen (1983), que dan cuenta de cómo la ansiedad interviene como "filtro afectivo" en el aprendizaje de nuevas lenguas; si a ello se añade un contexto desconocido, esta situación se agrava. Concretamente, desde el campo de la sociolingüística nos llegan aportaciones muy interesantes para nuestra labor docente relacionadas con el componente emocional de la lengua. En nuestro contexto investigaciones previas han puesto de manifiesto que las actitudes interculturales son un factor esencial en la educación intercultural, así como las actitudes lingüísticas lo son para el aprendizaje lingüístico. Vila (2006) recoge las aportaciones de Lambert, autor que hace ya varias décadas planteó la existencia de diferentes tipos de bilingüismo, relacionados con las actitudes lingüísticas del hablante y con la percepción de la importancia que concedía el contexto a su lengua materna como recurso para el aprendizaje de la nueva lengua. Considerando esta influencia, la promoción de la diversidad linguística exige a la escuela hacer visibles las lenguas maternas de nuestro alumnado y el reconocimiento de la pluralidad lingüística como un valor ya universal, como ha reconocido el Consejo de Europa.

\subsection{LAS LENGUAS COMO INSTRUMENTO DE COMUNICACIÓN Y DE ACCESO AL CURRÍCULUM}

En las situaciones de migración, el conocimiento de la lengua del país al que se llega es un elemento esencial para desenvolverse en el nuevo contexto. Un conocimiento que va mucho más allá de lo lingüístico lleva implícita una visión del mundo, determinadas formas de comunicar, etc. Cuando la nueva lengua se necesita no solo para la comunicación cotidiana, sino en un contexto escolar, es preciso conocer el lenguaje científico, textual, y los códigos orales y escritos de docentes y estudiantes, etc., es decir, incluir también la dimensión pragmática e intercultural.

Por eso, como señalamos anteriormente, una cuestión prioritaria es cómo ayudar al alumnado a desarrollar la competencia comunicativa en el ámbito escolar y nos referiremos al aspecto vinculado con las diferentes áreas (aunque no consideramos que todo el profesorado sea docente de lengua), dado que conocer la lengua de la escuela es una herramienta fundamental para acceder al currículum (Grupo Eleuterio Quintanilla, 2013) $\mathrm{y}$, por ende, a la equidad.

Desde un enfoque intercultural se contemplan diferentes dimensiones de las lenguas, entre otras: como vehículo de aprendizajes curriculares, desde el plurilingüismo, desde la competencia comunicativa intercultural. Para que se recojan las tres dimensiones, de nuevo es necesario insistir en un análisis de los factores contextuales en los que se va a desarrollar el currículum (alumnado destinatario, factores sociolingüísticos...), organización (tiempos, espacios...), recursos (cualificación docente, materiales, etc.).

La dimensión intercultural en el campo de las lenguas se ha ido desarrollando a partir de documentos como el Marco Común Europeo de Referencia para las Lenguas (MCER) (Instituto Cervantes, 2002), que hace alusión a la "consciencia intercultural" como:

el conocimiento, la percepción y la comprensión de la relación entre el "mundo de origen" y el "mundo de la comunidad objeto de estudio" (similitudes y diferencias 
distintivas) (...) que se enriquece con la conciencia de una serie de culturas más amplia que la que conlleva la lengua materna y la segunda lengua, lo cual contribuye a ubicar ambas en su contexto (...) (p. 99).

Dicha "consciencia" pertenece al ámbito declarativo, de observación, reflexión, pero es precisa la acción, la interacción entre personas de diferentes contextos culturales. Así, autores como Byram (1992; 1995), defienden la competencia comunicativa intercultural, que sería la habilidad para comunicarse de forma adecuada y flexible en un contexto multicultural. Implica diferentes competencias: cognitiva (conocimientos); afectiva (emociones y actitudes); comportamental (habilidades verbales y no verbales). Por tanto, se pasa de un enfoque lingüístico al comunicativo y al de interacción efectiva con personas de diferentes lenguas y culturas.

Como sabemos, tener fluidez conversacional no significa tener competencia comunicativa en la lengua académica para seguir el currículum, la denominada CALP (Cognitive Academic Language Proficiency) que como señala Vila (2011):

se asocia con el conocimiento lingüístico conceptual y constituye la dimensión de la competencia lingüística relacionada con las habilidades cognitivas y académicas (vocabulario poco familiar, producción de sintaxis compleja...). La competencia lingüístico cognitiva [...] se refiere a aspectos cognitivos como el conocimiento conceptual (comprensión de conceptos abstractos...), la capacidad metalingüística, el uso descontextualizado del lenguaje (p. 79).

De ahí que se hayan desarrollado diferentes programas, entre los que se encuentran el SIOP (The Sheltered Instruction Observation Protocol), o el AICLE (Aprendizaje Integrado de Contenidos y Lenguas Extranjeras). Este último tiene diferentes modalidades: a) los contenidos curriculares se dan en clase de lengua; b) en las clases de las diferentes materias se tienen en cuenta las dificultades lingüísticas del alumnado y se prevén los apoyos necesarios; c) enseñanza articulada lengua-contenidos: se coordinan los contenidos curriculares y los lingüísticos (Brinton, citado en Trujillo, 2005). También se han planteado otras propuestas, entre otras, las que conciben la lengua en términos de discurso de las diferentes áreas (p. 79).

Otro aspecto relevante está en el plurilingüismo. Es interesante realizar un análisis sociolingüístico del entorno (lenguas oficiales y de la comunidad, lenguas extranjeras, lenguas maternas del alumnado extranjero, etc.) y definir su papel en la escuela (de acceso al currículum, como mediación lingüística, etc.). Algunos intentos se han llevado a cabo en este sentido con los Proyectos Lingüísticos de Centro, pero que requieren un mayor desarrollo tanto en lo referente a las lenguas como a las diferentes materias.

Entre las cuestiones que propician el desarrollo de un currículum que favorezca el uso de las lenguas desde un planteamiento que facilite la equidad y la interculturalidad podríamos señalar:

- Buscar los aspectos de la lengua que comparten las diferentes disciplinas y las transferencias entre ellas, así como el componente intercultural que sea transversal a las mismas.

- Propiciar un desarrollo integrado de todas las lenguas del contexto y sus conexiones con las diferentes áreas del currículum. 
- cuestiones más concretas del día a día en el aula: Adaptar el habla del profesorado con buena articulación para dar explicaciones e instrucciones claras, proponer tareas muy estructuradas, ofrecer apoyos gráficos, palabras clave, etc.

- Propiciar situaciones de interacción en pequeños grupos de estudiantes que faciliten clarificar ideas, compartir conocimientos y visiones del mundo.

Por todo ello, queremos detenernos en algunas sugerencias sobre el papel que pueden desempeñar las lenguas maternas del alumnado extranjero en el aula, porque, como dice Unamuno (2003): "la gran homogeneidad cultural y lingüística que se está produciendo nos presenta la uniformidad como natural, cuando lo natural es, como decíamos, la diversidad y la variación" (p. 18).

\subsubsection{Contenidos y lenguas maternas}

Tener en cuenta la relación entre aprendizaje lingüístico y éxito/fracaso escolar orienta a replantearse el para qué se aprende (Trujillo, 2007; Vila, 2006), el qué se aprende y el cómo se aprende. Tal como se desprende de las aportaciones de ambos autores, la idea de un currículo basado en el desarrollo de competencias supone superar la concepción más tradicional centrada en el dominio del sistema lingüístico como un fin en sí mismo para pasar a un enfoque centrado en las funciones personales y sociales de la lengua.

En los contenidos, como venimos señalando, sería preciso realizar cambios profundos en la selección de los mismos y añadir nuevas perspectivas, incorporando desde todas las áreas aspectos que ayuden a comprender el mundo, la búsqueda, selección y tratamiento de la información a través de diferentes tipos de textos y en los que la información clave puede estar en las diferentes lenguas de la clase y difundirse mediante carteles para el aula, el centro y la comunidad educativa en general. Desde el punto de vista de la metodología, lo anterior entronca con el enfoque multilingüe y la necesidad de establecer andamiajes. Como vienen señalando algunas investigaciones (Cummis, 2007, p. 1), “debemos explorar las estrategias de enseñanza en el aula que ayudan a los estudiantes a transferir el conocimiento que tienen desde su propia lengua a la L2". Este autor ha demostrado, en su ya conocida Hipótesis de la Interdependencia Lingüística que, cuanto más competente se es en el conocimiento de la lengua materna más competente se será en la adquisición de otras lenguas. Si añadimos que, para el dominio de una nueva lengua hace falta un tiempo variable (pero que oscila, como mínimo, entre los 3 y los 5 años) y que existe cierta diferencia entre lo que se podría llamar "lenguaje conversacional" y un "lenguaje académico", podemos considerar las siguientes implicaciones de cara a nuestro trabajo como docentes:

- Si el alumnado dispone de conocimientos previos sobre el tema los transfiere con facilidad y afianza conceptos, que puede fijar mediante esquemas o borradores en su lengua materna, y si tiene que adquirirlos, el apoyo en su lengua materna le resultará valioso para lograrlos.

- Puede ser una oportunidad para que las familias se impliquen, al poder apoyarse en la lengua materna.

- Utilizar las lenguas maternas aumenta la autoestima del alumnado. Algo que en algunos momentos pueden considerar una carencia o poco prestigioso, se vuelve un elemento de reconocimiento, riqueza cultural y conocimiento de "otros". 
- Posibilita desarrollar una mayor conciencia y respeto por la diversidad lingüística y cultural del mundo.

- Es fundamental ser flexibles en la organización del tiempo en el aula, pues este alumnado puede requerir un intervalo mayor para poder transferir lo elaborado en su lengua materna a la nueva lengua.

- Es muy importante disponer de diccionarios en varias lenguas en el aula que faciliten la comprensión.

Ampliando las cuestiones relevantes sobre la metodología, es imprescindible tener en cuenta los estilos de aprendizaje y la cultura pedagógica y académica de procedencia. Si el alumnado está habituado al estudio individual y memorístico, familiarizarse con determinadas prácticas pedagógicas, requiere un tiempo. El enfoque de aprendizaje basado en problemas o en proyectos articulados de forma interdisciplinar o transdisciplinar, implicando a profesorado de diferentes áreas, que incorporen textos de identidad y fondos de conocimiento, enfoque multilingüe con las lenguas maternas como apoyo, como ComunicARTE, (Francos, 2014), puede ser un enfoque muy valioso para abordar cuestiones fundamentales que atañen a todos los seres humanos y a la vez, trabajar la interdependencia, asunto esencial para entender la construcción de la interculturalidad. Fomentar metodologías interactivas, de trabajo dialógico y colaborativo, ayudarán, igualmente, a crear un ambiente acogedor.

\subsubsection{Otra evaluación comunicativo-lingüística para un currículum intercultural}

Ya mencionamos la evaluación en nuestro currículum intercultural en los apartados anteriores. Es un ámbito complejo pendiente de un desarrollo matizado y eficaz. Además de lo ya comentado con carácter general, queremos añadir, específicamente para la evaluación del aprendizaje y dominio lingüísticos, que una de las cuestiones a abordar es la existencia de pruebas que sean comprensibles para todo el alumnado. Si nos referimos a la competencia comunicativa en una nueva lengua, en este momento se cuenta con pruebas estandarizadas de competencia en L2, para un perfil de población familiarizada con la cultura occidental, pero el alumnado de L2 es muy heterogéneo. Se han elaborado nuevas pruebas teniendo en cuenta no solo el público adulto, sino destinadas a alumnado adolescente (como las pruebas DELE, Diploma de español lengua extranjera, para escolares), pero con el mismo perfil. Según diferentes investigaciones (Genesee, Lindholm-Leary, Saunders y Christian, 2005) hay que tener en cuenta que el dominio de lectura y escritura puede ser muy diverso; por otra parte, es primordial tener en consideración que no parten del mismo conocimiento del mundo ni de los mismos referentes.

Si nos centramos en la evaluación escolar, en la que el alumnado tiene que dar cuenta de saberes académicos, la cuestión se complica aún más, pues debe contar con una buena competencia de lectura, comprensión y escritura, además de conocimientos de las diferentes materias según el currículum del país al que llega; así como comprender qué tipo de evaluación se plantea: memorística, por competencias, etc. La evaluación que defendemos, como ya señalamos, es la que contribuye a fomentar el aprendizaje autorregulado, la corresponsabilidad y la autonomía.

Uno de los instrumentos posibles para desarrollar otro tipo de evaluación a la existente es el Portfolio Europeo de las Lenguas (PEL) (Consejo de Europa, 2001), que permite 
recopilar información que se va generando en el proceso de aprendizaje, con la peculiaridad de que se seleccionan documentos significativos que permiten reflexionar sobre el trabajo realizado, a la vez que muestra la diversidad de cada individuo. Diferentes autores señalan su interés, entre otros, Cassany (1999), quien también destaca sus características: el estudiante es protagonista del aprendizaje; se orienta a los procesos; es interdisciplinar y es flexible porque integra diferentes metodologías y permite evaluar de otro modo.

También podemos añadir (Francos, 2011) que recoge los conocimientos adquiridos previamente y fuera del contexto escolar (familia, experiencia vital, etc.). Son especialmente útiles los apartados de biografía lingüística y dossier. Este último posibilita conocer el aprendizaje realizado con diferentes tipos de textos (testimonios, gráficos, cronologías...), y de procedimientos seguidos (definir conceptos, consulta de fuentes primarias...). De este modo se propician aprendizajes interdisciplinares y autónomos.

\section{A MODO DE REFLEXIÓN FINAL}

Con este artículo hemos querido retomar una reflexión crítica sobre el currículum, especialmente desde el enfoque intercultural. Este enfoque implica una visión dinámica, que nos invite a observarnos críticamente como docentes, en el uso que hacemos de la diversidad sociolingüística de nuestro entorno, del tipo de evaluación que llevamos a cabo, qué contenidos y qué metodologías forman parte de nuestra práctica docente. No se trata de elaborar aquí un documento del currículum, pero sí insistir en los cambios posibles, en cómo es posible elaborar un proyecto de centro que puede ser multilingüe y multidisciplinar, con una mirada más diversa. En definitiva, para elaborar un currículum inclusivo e intercultural, tal como se viene defendiendo desde diferentes ámbitos desde los últimos años, es necesario recoger todas las reflexiones teóricas del campo de lenguas, de la sociología, la pedagogía, las didácticas... que plantean una mirada diversa, establecer puentes entre las diferentes materias y reformular los contenidos, las metodologías, la organización de tiempos y espacios, la evaluación. Es decir, diseñar un proyecto educativo de centro (PEC) que incluya la variable intercultural como línea definitoria de la organización del centro, de la práctica del aula y del trabajo con el entorno comunitario.

\section{REFERENCIAS BIBLIOGRÁFICAS}

Abajo, J. E. y Carrasco, S. (2011). La situación escolar del alumnado de minorías étnicas: el modelo explicativo ecológico-cultural de John Ogbu, Recerca, Revista de Pensament y Análisi, 11, 7192.

Apple, M.W. y Beane, J. A. (2005). La defensa de las escuelas democráticas. En M.W. Apple y J.A. Beane (pp. 13-47), Escuelas democráticas (4 edición). Madrid: Morata.

Ávila, M., Calatayud, Mª́., Cantón, I., Castillo, S. y Zaitegui, N. (2010). La evaluación como proceso sistemático para la mejora. Colección: Conocimiento Educativo. Serie: Aula Permanente. Madrid: IFIIE.

Bauman, Z. (2003). Comunidad. En busca de seguridad en un mundo hostil. Madrid: Siglo XXI.

Byram, M. (1992). Culture et éducation en langue étrangère. París: Hatier.

Byram, M. (1995). Intercultural Competence and Mobility in Multinational Contexts: a European View. Clevedon, UK: Multilingual Matters. 
Estudios Pedagógicos XLVI Nº 1: 431-450, 2020

EDUCACIÓN INTERCULTURAL: ALGUNAS REFLEXIONES Y ORIENTACIONES PARA UNA PRÁCTICA PEDAGÓGICA EN UNA ESCUELA DEMOCRÁTICA Y PLURAL

Besalú, X. (2008). Educar en sociedades pluriculturales. Barcelona. Cuadernos de Pedagogía: Biblioteca Básica para el profesorado. (marzo, 2010). La educación intercultural y el currículo escolar. Congreso Internacional en la Red sobre interculturalidad y educación.1-21. Recuperado de: https://aulaintercultural. org/2010/02/26/la-educacion-intercultural-y-el-curriculo-escolar

Bolívar, A. (2004). Ciudadanía y escuela pública en el contexto de diversidad cultural. Revista Mexicana de Investigación Educativa, 9(20), 15-38.

Calatayud, M.A. (2018). Los agrupamientos escolares a debate. Tendencias Pedagógicas, 32, 5-14.

Cassany, D. (1999). Construir la escritura. Paidós: Barcelona.

Circular de 14 de noviembre de 2017 sobre la atención educativa al alumnado de incorporación tardía. Recuperado de: https://www.educastur.es/-/circulares-alumnado-incorporacion-tardiacurso-2017-20

Circular sobre la atención educativa al alumnado con incorporación tardía al sistema educativo español para el curso 2018-2019. Recuperado de: https://www.educastur.es/-/circularesalumnado-incorporacion-tardia-curso-2018-2019

Consejo de Europa (2001). Portafolio Europeo de las Lenguas Recuperado de: http://sepie.es/doc/ newsletter/2017/febrero/NOTICIA_PEL.pdf

Consejería de Educación y Cultura del Principado de Asturias y CIDE (2003). La atención a alumnos inmigrantes en la Comunidad Autónoma de Asturias. Recuperado de: https://docplayer. es/85860318-La-atencion-a-alumnos-inmigrantes-en-la-comunidad-autonoma-de-asturias.html

Cummis, J. (2007). Promoting literacy in multilingual contexts. Research monograph, 5. Recuperado de: http://www.edu.gov.on.ca/eng/literacynumeracy/inspire/research/cummins.pdf

Díez-Gutiérrez, E.J. (2014). La práctica educativa intercultural en Secundaria. Revista de Educación, $363,12-34$.

Díaz-Aguado, M.J. (2004). Educación intercultural y cooperación. Una nueva interacción educativa para un mundo que también es diferente. Educatio, 22, 59-89.

Escarbajal, A. (2010). La escuela inclusiva en una sociedad pluricultural y la importancia del trabajo colaborativo. Enseñanza \& Teaching, 28(2), 161-179.

Esteban, M. (2011). La geografía vital y psicológica de la interculturalidad. Cuadernos Interculturales, 9(16), 33-44.

Esteban, M. y Vila, I. (2013). CM 22 experiencias en la educación inclusiva: vinculación familia, escuela y comunidad. Barcelona: Horsori.

Fernández Enguita, M. (2001). Educar en tiempos inciertos. Madrid: Morata.

Francos, C. (2011). El PEL como instrumento de evaluación de áreas no lingüísticas. En I. Ballano, (Coord.). La evaluación en el aprendizaje de lenguas por inmigrantes. Bilbao: Universidad de Deusto.

(2014). Proyecto Comunicarte: Arte y palabras para aprender español y participar en la ciudad. Una propuesta didáctica. Didáctica. Lengua Y Literatura, 26, 169-196. https://doi. org/10.5209/rev_DIDA.2014.v26.46838

Genesee, F., Lindlholm-Leary, K., Saunders, W. and Christian, D. (2005). English Language Learners in US Schools: An Overview of Research Findings. Journal of Education for students placed at risk, 10(4), 363-385.

Gil-Jaurena, I. (2013). Educación intercultural y animación socio-cultural: convergencias y reflexiones. Revista Iberoamericana de Educación, 61(4), 1-7. Recuperado de: https://doi.org/ https://doi.org/10.35362/rie614924

Gimeno, J. (1996). Cambiar los contenidos, cambiar la educación. Madrid: Morata.

Grupo Eleuterio Quintanilla (1996) Materiales para una educación antirracista. Educación antirracista y diversidad cultural en el Estado Español. Madrid: Talasa.

Grupo Eleuterio Quintanilla. (2000). Educación Intercultural: Por la diversidad cultural y la igualdad social. Trabajadores de la Enseñanza, suplemento enero. 
Grupo Eleuterio Quintanilla (2003). Alumnado extranjero en la escuela asturiana. Medidas urgentes para una educación de calidad. Grupo Eleuterio Quintanilla, CCOO enseñanza, FETE-UGT, SUATEA.

Grupo Eleuterio Quintanilla (2013). Entre Palabras y Voces. Propuestas para la enseñanza de la lengua de instrucción desde un enfoque intercultural e inclusivo. Gijón: Letra 25.

Instituto Cervantes (2002). Marco común europeo de referencia para las lenguas: aprendizaje, enseñanza, evaluación. Madrid: MECD-Anaya. Recuperado de: http://cvc.cervantes.es/ ensenanza/biblioteca_ele/marco/

Huguet, A. \& Navarro, J. (2006). Inmigración y escuela. Revista interuniversitaria de formación del profesorado, (56), 17-22.

Jiménez, R.A., Sánchez, C. A., García, Ma . C. y Cotrina, M. (2011). Explorar y comprender el fracaso escolar en el contexto multicultural de Ceuta. Respuestas de la institución escolar. En F.J. García y N. Kressova, Actas del I Congreso Internacional sobre Migraciones en Andalucía (pp. 133147). Granada: Instituto de Migraciones.

Koustourakis, G.S., Asimaki, A. y Spiliopoulou, G. (2016). Cultural capital and educational expectations of native and inmigrant parents of Primary School students: qualitative study. Revista Internacional de Sociología de la Educación, 5(3), 165-189.

Krashen, S. D. y Terrel, T. D. (1983). The Natural Approach: Language Acquisition in the Classroom. Oxford: Pergamon.

López-Jiménez, Ma D. (2018). Diversidad y contenidos socio-culturales en libros de texto para la enseñanza del español como L2. Revista de Educación, 382, 11-31.

Louzao, M. (2015). Diversidad lingüística y educación intercultural. Propuestas de actuación en Educación Infantil. Revisa nacional e internacional de educación inclusiva, 8(2), 171-184.

. (2016). La educación intercultural que se avecina: nuevos retos, nuevas posibilidades. A.S. Jiménez et al (Coords.). Congreso Internacional e Interuniversitario contra la pobreza infantil en el mundo. Huelva 2015-Asunción 2016.

(2019). Educación intercultural: de la urgencia cuantitativa a la exigencia democrática. Orientaciones para la investigación y la acción en el contexto educativo asturiano. Revista de Investigación en Educación, 17(1), 44-54.

Massot, I. (2003). Jóvenes entre culturas. La construcción de la identidad en contextos multiculturales. Bilbao: Desclée de Brouwer.

Merchán, J. (2012). La introducción en España de la política educativa basada en la gestión empresarial de la escuela: el caso de Andalucía. Education Policy Analysis Archives, 20(32). Recuperado de: http://epaa.asu.edu/ojs/article/view/1059.

. (2013). Docentes, familias y alumnos en las políticas educativas neoliberales. Con-Ciencia Social, (17), 137-144.

Ministerio de Educación y Formación Profesional (2019). Datos y Cifras: Curso Escolar 2019-2020. Secretaría General Técnica. Subdirección General de Atención al Ciudadano. Documentación y Publicaciones. Recuperado de: http://www.educacionyfp.gob.es/servicios-al-ciudadano/ estadisticas/indicadores-publicaciones-sintesis/datos-cifras.html

Ogbu, J. U. (1993). Etnografía escolar: una aproximación a nivel múltiple, En A. Díaz de Rada, M. Velasco y F.J. García-Castaño (Coords.), Lecturas de antropología para educadores. El ámbito de la antropología de la educación y de la etnografía escolar, (pp. 145-174). Madrid: Ediciones Trotta.S.A.

Rodríguez-Izquierdo, R. M. (2009). La investigación sobre la educación intercultural en España. Archivos Analíticos de Políticas Educativas, 17(4), 1-29.

Romero, J. (2005). La co-participación de la escuela en la producción social de la infancia. Nota crítica sobre moratorias, desarrollo personal y crecimiento político. En P. Dávila y L. Ma Anaya (Coords.). La infancia en la historia: espacios y representaciones (15-426), Vol. II, XIII Coloquio de Historia de la Educación. San Sebastián: Espacio Universitario/EREIN. 
Estudios Pedagógicos XLVI N ${ }^{\circ}$ : 431-450, 2020

EDUCACIÓN INTERCULTURAL: ALGUNAS REFLEXIONES Y ORIENTACIONES PARA UNA PRÁCTICA PEDAGÓGICA EN UNA ESCUELA DEMOCRÁTICA Y PLURAL

(2018). La historia del currículum como fuente para la Didáctica de las Ciencias Sociales. Revista de Investigación en Didáctica de las Ciencias Sociales, 3, 103-118.

Schmelkes, S. (2018). Toward an intercultural approach to evaluation: a perspective from INEE in Mexico. Education Policy Analysis Archives, 26(52). http://dx.doi.org/10.14507/epaa.26.3818

Susinos, T. y Ceballos, N. (2012). Las posibilidades de la voz del alumnado para el cambio y la mejora educativa. Presentación. Revista de Educación, Monográfico sobre las posibilidades de la voz del alumnado para el cambio y la mejora educativa, (359), 16-23.

Torres Santomé, X. (2011). La justicia curricular. El caballo de Troya de la cultura escolar. Madrid: Morata.

Touraine, A. (2001). Igualdad y diversidad. Las nuevas tareas de la democracia. México: FCE.

Trujillo, F. (2005). La integración de lenguaje y contenidos en la enseñanza del español como segunda lengua en el contexto escolar: implementación y evaluación. Revista electrónica RedEle, 4. . (2007). Enseñar nuevas lenguas en la escuela: L1, L2, LE..., NL. Revista de Educación, $343,71-91$.

Unamuno, V. (2003). Lengua, escuela y diversidad sociocultural. Barcelona: Graó.

Verdeja, M. (2015). Aportaciones de la pedagogía de Paulo Freire a la educación intercultural. Posibilidades de aplicación al sistema educativo en Asturias. [Tesis Doctoral]. Oviedo: Universidad de Oviedo.

Verdeja, M. y González. X.A. (2018). Aportaciones de Paulo Freire a la educación intercultural. Un estudio de caso en la Educación Secundaria Obligatoria. Revista Iberoamericana de Educación, 76(1), 143-168.

Vila, I. (2006). Adquisición de lenguas extranjeras y competencia multilingüe. Jornadas Pedagógicas del Programa de Lengua y Cultura Portuguesa. Mérida, 15-17 noviembre.

. (2011). La evaluación del alumnado extranjero y su conocimiento lingüístico. En I. Ballano, (coord.) La evaluación del alumnado inmigrante. Bilbao: U. de Deusto.

Walsh, C. (2010). Interculturalidad crítica y educación intercultural. En J. Viaña, L. Tapia y C. Walsh (eds.). Construyendo Interculturalidad Crítica (pp. 75-96). La Paz: III-CAB. 